\title{
Semantic Based Substitution of Unsupported Access Points in the Library Meta-search Environments
}

\author{
Michalis Sfakakis and Sarantos Kapidakis \\ Laboratory on Digital Libraries \& Electronic Publishing, \\ Department of Archive \& Library Sciences, Ionian University \\ 72, Ioannou Theotoki Str., Corfu, Greece, GR-49100 \\ \{sfakakis, sarantos\} @ionio.gr
}

\begin{abstract}
Meta-searching library communities involve access to sources where metadata are invisible behind query interfaces. Many of the query interfaces utilize predefined abstract Access Points for the implementation of the search services, without any further access to the underlining meta-data and query methods. The existence of unsupported Access Points and its consequences, which are either query failures or inconsistent query answers, creates a major issue when meta-searching this kind of systems. An example of the abstract Access Point based search model is the Z39.50 information retrieval protocol, which is widely used by the library community. In this paper we present the zSAPN (Z39.50 Semantic Access Point Network), a system which improves the search consistency and eliminates the query failures by exploiting the semantic information of the Access Points from an RDFS description. The current implementation of zSAPN is in the context of the Z39.50 protocol, using the official specification of the Access Point semantics and can benefit the huge number of the available sources worldwide. zSAPN substitutes each unsupported Access Point with a set of other supported ones, whose appropriate combination would either broaden or narrow the initial semantics, according to the user's choice. Finally, we estimate the impact of the modification of the initial semantics during the substitution process to the precision or the recall of the original query, with the unsupported Access Point.
\end{abstract}

\section{Introduction}

In recent years, meta-search engines allow users to access a number of distributed and autonomous metadata repositories. These information sources are hidden behind query interfaces without any access to the underlying data structures and query methods. In some cases query methods for the underlying sources share common characteristics or conform to a standard information retrieval protocol. This is mostly the case when metadata describe materials from a common information community. One significant task affecting the effectiveness of the meta-search engine is its ability to transform the user's query into every underlying source's query system.

Moreover, in the library community, many information sources publicize their metadata with query interfaces based on the concept of the abstract Access Point and mainly conforming to the Z39.50 information retrieval protocol. The variant or poor

B. Christensen-Dalsgaard et al. (Eds.): ECDL 2008, LNCS 5173, pp. 296-307, 2008.

(C) Springer-Verlag Berlin Heidelberg 2008 
implementations of the protocol restrict the query interfaces with a relatively small number of available Access Points. Unsupported Access Points are very common, especially when searches are addressed to more than one sources. When a query contains an unsupported Access Point the query fails or generates inconsistent answer if the source arbitrarily substitutes the unsupported Access Point with a supported one.

The common approaches for dealing with the unsupported Access Points in order for the user to receive partial results instead of getting query failure, are either to permit queries with only the common Access Points to all sources, or to ignore the sources that do not support the Access Point, or finally to allow the source to substitute the unsupported Access Point with a supported one. The first approach restricts the search capabilities of the sources, the second restricts the available sources, while the third produces unpredictable answers.

zSAPN (Z39.50 Semantic Access Point Network) mediator system component attacks the problem of the unsupported Access Points, in the context of the Z39.50 protocol and for the Access Points specified into the Bib-1 attribute set, by substituting each unsupported Access Point with the union or the intersection of others supported. The substitution exploits the semantics of the Access Points from an RDFS [10] description, and according to the user preferences either broadens or narrows the semantics of the original unsupported Access Point. When substituting the unsupported Access Point we avoid the failure of the query. Furthermore, and of greater importance, the presented version of zSAPN estimates how the combination of the selected Access Points for the substitution affects the precision and the recall of the original query with the unsupported Access Point. Therefore, a user could better reformulate a query through an iterative process and based on feedback on the modification of the recall or the precision of the original query. Moreover, we are able to discover if a substitution produces a query with equivalent semantics to the original, or we can better schedule the retrieval of the metadata records in order to make a more efficient post processing of them, if it is desirable. The estimation is based on measures for the taxonomic similarity of concept hierarchies used in ontology learning process $[4,6]$.

The remaining sections of this study are presented as follows: section 2 outlines the concept of the abstract Access Point and the access methods of Z39.50 information retrieval protocol. Thereafter, the section describes the semantics based access point network in the context of Z39.50 and its extended RDFS description. In Section 3 we describe the substitution policies and their taxonomic recall and precision evaluation measures. Section 4 presents the zSAPN and puts forward many interesting remarks while searching some representative world wide popular sources. Finally, section 5 concludes and presents a number of interesting points for further research and improvements of zSAPN.

\section{Access Points and the Z39.50 Protocol}

\subsection{Access Points}

In a catalogue or a Database, an access point is any part of the record under which the record may be searched, identified and retrieved [3]. A name, a term, a heading, or a 
code, etc, are some examples of possible access Points. From the information system point of view, these parts of the records are grouped together according to some criteria and generate abstract (or virtual) indexes for searching the catalogue or the database. It is very common to refer to these virtual indexes as abstract Access Points. Moreover, any Access Point (abstract or concrete) has a 'denotational' semantics (what it describes) and an 'operational' semantics (what implications does it have for query evaluation). In this work we use the term of the Access Point as it is used in the context of the information system.

\subsection{The Z39.50 Protocol}

The Z39.50 client/server information retrieval protocol [1] is widely used in library electronic communication for searching and retrieving information from a number of diverse, distributed, heterogeneous and autonomous sources, not necessarily with the same structure and search capabilities. The query mechanism of the Z39.50 protocol utilizes sets of predefined Access Points combined with specific attributes (i.e. Attribute Sets) in a number of different query language specifications (i.e. query types). For the accomplishment of the standard search primitives the general conformance requirements of the protocol specify that at least the Access Points defined in the attribute set Bib-1 and the query Type-1 for the query formulation have to be recognized (although not necessarily implemented).

According to the protocol, if a source does not support an Access Point, the source should fail the search (i.e. query failure) and supply an appropriate diagnostic message, or the source will substitute it according to the requested 'Semantic Action' attribute value. The MELVYL union catalogue, the COPAC Academic \& National Library Catalogue (UK) and the Library and Archives Canada are some international sources which follow the former approach, while the Library of Congress is a representative of the latest approach. It is worth mentioning that the Z39.50 source of the Library of Congress does not provide any information concerning the substitution of the unsupported Access Point.

A common question concerning the unsupported Access Points is how often unsupported Access Point occur? Statistical figures -provided by IndexData ${ }^{1}$ - present- $^{-}$ ing the "Ten most commonly supported Access Points" indicate that no single Access Point is universally supported by Z39.50 sources. From a set of 2,869 sources tested where 1,821 of them support the search service, the most commonly supported Access Point is the Title $(1,667$ or $91.54 \%)$ and then is the Subject heading $(1,634$ or $89.73 \%$ ) followed by the Author (1,629 or $89.45 \%)$.

\subsection{Z39.50 Semantics Access Point Network}

The official semantics of the Z39.50 Bib-1 Access Points are defined in [11], which represents a consensus among the members of the Z39.50 Implementors Group (ZIG). The document defines the semantics of the Access Points using tag values of representative MARC bibliographic format fields. As an example, the definition for the

\footnotetext{
${ }^{1}$ http://targettest.indexdata.com/stats.html. The Z39.50 servers were last tested on Friday $17^{\text {th }}$ of March 2008.
} 
Access Point Author-name-conference includes the data from the fields with MARC Tags $\{111,411,711,811\}$.

We semantically associate the Access Points using their subset relationships. We consider an Access Point to be a subset of another if the set of the data fields used to create the first one is a subset of the set of the data fields used to create the second one. As an example, consider the Access Point Author-name which, according to its definition [11], includes the data from the fields with MARC tags included in the set $\{100,110,111,400,410,411,700,710,711,800,810,811\}$, and also, the Access Point Author-name-conference which includes the data from the set of fields $\{111$, 411, 711, 811 \}. The Access Point Author-name-conference is considered as being a subset of the Author-name.

It is worth mentioning that parts of the metadata records (fields, subfields, etc.) that are used to generate an abstract Access Point could also be considered as Access Points too. Thereafter, an abstract Access Point is a superset of every Access Point representing any constitutional part of the abstract Access Point from the metadata record. Therefore, using the previous example, the Access Point representing the field with tag 100 could also be considered as a subclass of the abstract Access Point $A u$ thor-name.

We represent the relationships between the Access Points with a directed graph G in which the nodes represent Access Points and the arcs represent subset relationships. This graph has an arc $<i, j>$ if and only if Access Point $i$ is a subset of Access Point $j$. The Access Points Author-name and Author-name-conference, used in the previous example, will be represented by two nodes of the graph and their subset relationship from the arc <Author-name-conference, Author-name $>$. The automated process of the construction of the Semantics-Based RDF Schema of the Bib-1 Access Points is based on our previous work described in [7]. The presented extended version of the RDFS includes both the Bib-1 abstract Access Points with their subset Access Points representing their constitutional parts. A main feature of the graph is that leaf nodes (i.e. classes without subclasses) correspond to Access Points representing the constitutional parts of abstract Access Point.

For the construction of the Bib-1 Access Points RDF Schema, we map the nodes of the graph to rdfs:Class and the arcs of the graph to rdfs:subClassOf properties. Figure 1 depicts a representative sample of the resulting RDFS graph. The complete version of the graph can be found at http://dlib.ionio.gr/lab/graph.htm.

The similarity of the semantics of an Access Point with others is expressed from their positions in the graph. The direct subclasses or super classes of an Access Point have the nearest semantics with the class which represents the Access Point. Furthermore, the semantics of an Access Point produced from the union or the intersection of a set of Access Points are assigned from the union or the intersection of the corresponding constitutional access points sets. Let's consider the Access Point Authorname-conference and the set of Access Points \{Author-name, Name-conference $\}$. From figure 1 we see that the intersection of the constitutional access points sets for the Access Points Author-name and Name-conference is the set \{mrc:f-111, mrc:f411, mrc:f-711, mrc:f-811\} which specifies the semantics of the Access Point Authorname-conference. Finally, an Access Point has equivalent semantics with another Access Point if the sets of their underlying constitutional Access Points are equal. 


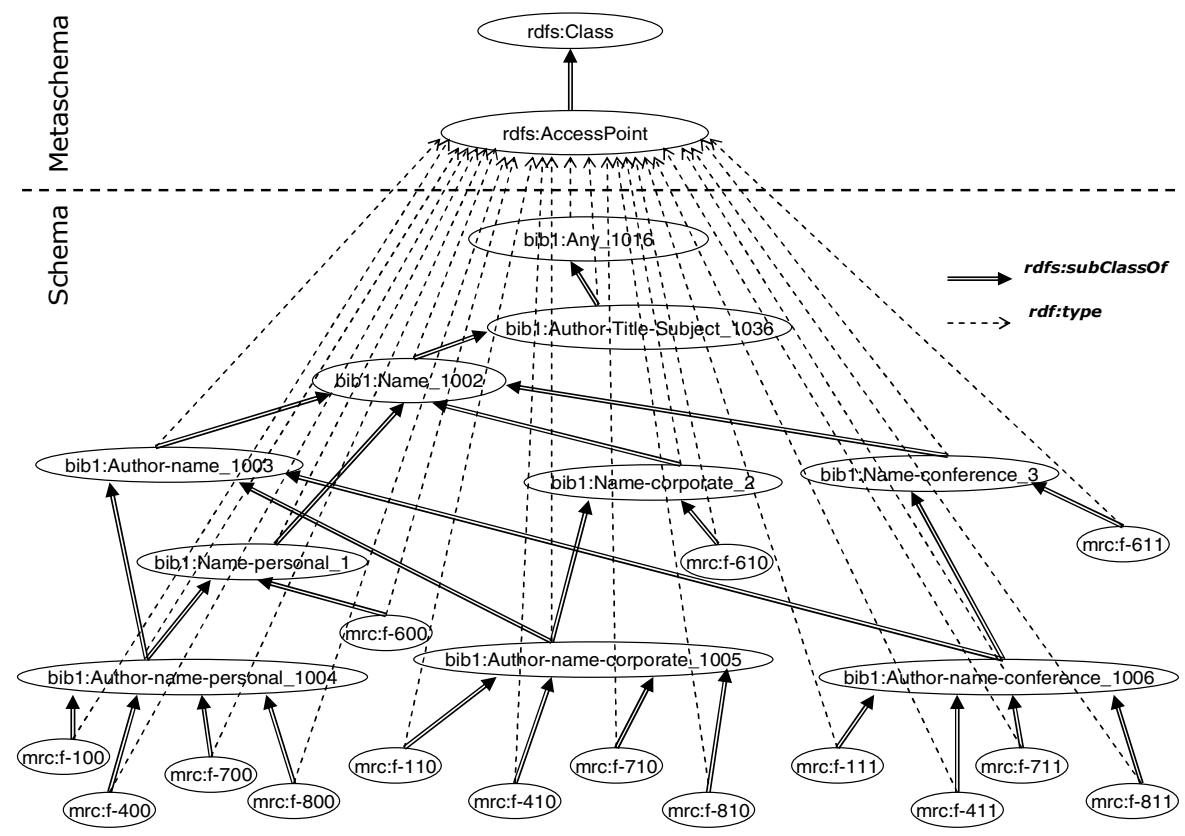

Fig. 1. A representative sample of the Semantics-Based RDFS of the Bib-1 Access Points

The similarity among the semantics of the Access Points influences the result sets of the queries with the Access Points. Thus, when a query uses an Access Point near the top of the hierarchy, the answer contains more results than a query contains using an Access Point near the leafs of the hierarchy. Consequently, an ancestor Access Point of another broadens the semantics of the Access Point and while lowering the precision increases the resulting hits in the answers. In contrast, a descendant of an Access Point narrows the semantics of the Access Point and while lowering the recall decreases the resulting hits in the answers.

\section{Access Point Substitution}

\subsection{Broad and Narrow Substitution Policies}

Exploring the Access Points similarity from the Bib-1 RDFS, zSAPN provides two alternative substitution policies for the unsupported Access Point. The formal description of the substitution methods and the algorithms are described in our previous work [8]. The first policy broadens the semantics of the unsupported Access Point and substitutes it with the Boolean AND combination of all the nearest supported ancestors of the Access Point, from every ancestor path hierarchy starting from the unsupported Access Point. The final set of Access Points from the broad substitution policy is the minimal set of supported Access Points having the nearest semantics to the unsupported Access Point, after an optimization step. This is the case when there are more 
than one ancestor path hierarchies containing a supported Access Point, while the selected Access Point from one path is also a member of another path at a higher level position than the selected AP from this path.

In contrast, the second policy narrows the semantics of the unsupported Access Point and substitutes it with the Boolean $O R$ combination of all the nearest supported descendants of the Access Point, from every descendant path hierarchy starting from the unsupported Access Point. Similarly, as for the broad substitution is, the final set of Access Points from the narrow substitution policy is the minimal set of supported Access Points having the nearest semantics to the unsupported Access Point, after an optimization step. This is the case when there are more than one descendant path hierarchies containing a supported Access Point, while the selected Access Point from one path is also a member of another path at a lower level position than the selected Access Point from this path.

As it is discussed in the next section, broad substitution decreases the precision of the resulting query without affecting the recall, while the narrow substitution decreases the recall of the resulting query without affecting the precision. Therefore, when both substitution policies are feasible, one substitution could be chosen over to another according to the user preferences over the recall or the precision.

\subsection{Precision and Recall Evaluation}

The broad substitution decreases the precision of the resulting query without affecting the recall. This is due to the fact that the substituting access points with super classes Access Points match the query term against all existing fields in the requested Access Point plus some others. Therefore, in order to evaluate the decrease of the precision we adapt the taxonomic precision measure $(t p)$ from the ontology learning framework described in $[4,6]$, using the characteristic extract leaf subclasses. While the general case for the taxonomic precision measure has to compare two concepts from two different ontologies, the gold or reference and the retrieved or learned, our evaluation has to compare two concepts from the same ontology, represented from the Z39.50 Semantic Access Point Network RDFS.

Considering that our ontology $O$ represents the RDFS graph for the Z39.50 Semantic Access Point Network as described in section 2.3, and that $C$ is the set of all concepts in $O$ representing the Access Points. Let $\leq_{C}$ be a relation on $C: c_{1} \leq_{C} c_{2}$ holds if $c_{1}$ is a subclass of $c_{2}$. With ${S_{C}}^{+}$we denote the transitive closure of $\leq_{C}$. We consider $c_{1}$ to be an ancestor of $c_{2}$ (or $c_{2}$ to be a descendant of $c_{1}$ ) if $c_{2} \leq_{C}{ }^{+} c_{1}$.

We define the set of leaf subclasses (lsc) of an Access Point as follow:

$$
l s c(a p, O)=\left\{a p_{i} \mid a p_{i} \in C \wedge a p_{i} \leq^{+} a p \wedge \neg \exists x \in C: x \leq a p_{i}\right\}
$$

It is worth mentioning that the leaf subclasses of an Access Point, which are also Access Points, correspond to the parts of the record that will be matched against the search term. Therefore, the leaf subclasses of an Access Point specify its semantics.

The taxonomic precision ( $t p$ ) of two Access Points $a p_{r}, a p_{s}$ where, $a p_{r}$ and $a p_{s}$ represent the requested and the selected for the substitution Access Points respectively is defined as:

$$
t p\left(a p_{s}, a p_{r}, O\right)=\left|l s c\left(a p_{s}\right) \cap l s c\left(a p_{r}\right)\right| / l s c\left(a p_{s}\right) \mid
$$


The taxonomic precision represents the proportion of the fields used into the requested Access Point (relevant fields) out of the fields used into the selected Access Point for the substitution (searched fields). It is noticeable that the $t p$ construct is not symmetric. Moreover, as every Access Point from the broad substitution policy belongs into an ancestor path hierarchy starting from the requested Access Point the $l s c\left(a p_{r}\right) \subseteq l s c\left(a p_{s}\right)$ property holds. Thereafter, a simplified form of the equation (1) for the taxonomic precision for an Access Point produced from the broad substitution policy is:

$$
\operatorname{tp}\left(a p_{s}, a p_{r}, O\right)=\left|l s c\left(a p_{r}\right)\right| / l s c\left(a p_{s}\right) \mid
$$

The final step of the broad substitution policy is the Boolean AND combination of the selected Access Points. Therefore, the derived access point set could be considered as a new abstract Access Point created from the common fields of the Access Points used in the Boolean AND combination. The taxonomic precision of the intersection of a set of Access Points $\left\{a p_{s l}, a p_{s 2}, \ldots, a p_{s n}\right\}$ produced from the broad substitution is defined from the next simplified formula:

$$
\operatorname{tp}\left(\cap a p_{s i}, a p_{r}, O\right)=\left|l s c\left(a p_{r}\right)\right| /\left|l s c\left(\cap a p_{s i}\right)\right|
$$

In contrast, the narrow substitution decreases the recall of the resulting query without affecting the precision. This is due to the fact that the subclasses of the Access Points match the query term against some of the existing fields in the requested Access Point. Also, we remind that the selected Access Point from the narrow substitution policy is a subclass of the requested, therefore $l s c\left(a p_{s}\right) \subseteq l s c\left(a p_{r}\right)$ and $l s c\left(a p_{s}\right) \cap l s c\left(a p_{r}\right)=l s c\left(a p_{s}\right)$ properties hold. Thereafter, the corresponding formulas for the taxonomic recall (tr) constructs are:

$$
\begin{gathered}
\operatorname{tr}\left(a p_{s}, a p_{r}, O\right)=\left|l s c\left(a p_{s}\right) \cap l s c\left(a p_{r}\right)\right| / l s c\left(a p_{r}\right) \mid \\
\operatorname{tr}\left(a p_{s}, a p_{r}, O\right)=\left|l s c\left(a p_{s}\right)\right| /\left|l s c\left(a p_{r}\right)\right| \\
\operatorname{tr}\left(\cup a p_{s i}, a p_{r}, O\right)=\left|l s c\left(\cup_{s i}\right)\right| /\left|l s c\left(a p_{r}\right)\right|
\end{gathered}
$$

Table 1 depicts representative taxonomic precision and recall values for some meaningful broad and narrow substitutions respectively (see figure 1). For both substitution policies, the first row represents the requested Access Point, while the first column represents the substituting Access Point. Thereafter, the upper right part from the diagonal of the table gives the taxonomic precision ( $t p$ ) values for some feasible broad substitutions. Observing the second row, we see that when Name_1002 substitutes its direct descendant Author-name_1003 the tp remains high (0.8), while the $t p$ lowers when moving to the end of the row where Name_1002 substitutes its descendant Author-name-personal_1004. Alike broad substitution, the lower left part from the diagonal of the table gives the taxonomic recall ( $t r)$ values for some feasible narrow substitutions. Observing now the second column, we see that when Authorname_1003 substitutes its direct ancestor Name_1002 the tr remains high (0.8), while tr lowers when moving to the end of the column where Author-name-personal_1004 substitutes its ancestor Name_1002 ( $t p=0.26)$. 
Table 1. Example of taxonomic precision and recall values for some meaningful broad and narrow substitutions respectively

\begin{tabular}{|c|c|c|c|c|c|c|c|c|}
\hline $\operatorname{tr}_{\operatorname{tr}}^{\operatorname{tp} \rightarrow}$ & 1002 & 1003 & 3 & 2 & 1 & 1006 & 1005 & 1004 \\
\hline Name_1002 & 1 & $\begin{array}{l}12 / 15 \\
=0.8\end{array}$ & $\begin{array}{l}5 / 15= \\
0.33\end{array}$ & $\begin{array}{l}5 / 15= \\
0.33\end{array}$ & $\begin{array}{l}5 / 15= \\
0.33\end{array}$ & $\begin{array}{l}4 / 15= \\
0.26\end{array}$ & $\begin{array}{l}4 / 15= \\
0.26\end{array}$ & $\begin{array}{l}4 / 15= \\
0.26\end{array}$ \\
\hline Author-name_1003 & $\begin{array}{l}12 / 15= \\
0.8\end{array}$ & 1 & & & & $\begin{array}{l}/ 12= \\
0.33\end{array}$ & $\begin{array}{l}4 / 12= \\
0.33\end{array}$ & $\begin{array}{l}4 / 12= \\
0.33\end{array}$ \\
\hline Name-conference_3 & $\begin{array}{l}5 / 15= \\
0.33\end{array}$ & & 1 & & & $\begin{array}{l}4 / 5= \\
0.8\end{array}$ & & \\
\hline Name-corporate_2 & $\begin{array}{l}5 / 15= \\
0.33\end{array}$ & & & 1 & & & $\begin{array}{l}4 / 5= \\
0.8\end{array}$ & \\
\hline Name_personal_1 & $\begin{array}{l}5 / 15= \\
0.33\end{array}$ & & & & 1 & & & $\begin{array}{l}4 / 5= \\
0.8\end{array}$ \\
\hline $\begin{array}{l}\text { Author-name- } \\
\text { conference_1006 }\end{array}$ & $\begin{array}{l}4 / 15= \\
0.26\end{array}$ & $\begin{array}{l}4 / 12= \\
0.33\end{array}$ & $\begin{array}{l}4 / 5= \\
0.8\end{array}$ & & & 1 & & \\
\hline $\begin{array}{l}\text { Author-name- } \\
\text { corporate_1005 }\end{array}$ & $\begin{array}{l}4 / 15= \\
0.26\end{array}$ & $\begin{array}{l}4 / 12= \\
0.33\end{array}$ & & $\begin{array}{l}4 / 5= \\
0.8\end{array}$ & & & 1 & \\
\hline $\begin{array}{l}\text { Author-name- } \\
\text { personal_1004 }\end{array}$ & $\begin{array}{l}4 / 15= \\
0.26\end{array}$ & $\begin{array}{l}4 / 12= \\
0.33\end{array}$ & & & $\begin{array}{l}4 / 5= \\
0.8\end{array}$ & & & 1 \\
\hline
\end{tabular}

\section{4 zSAPN Presentation}

The system zSAPN can be accessed at http://dlib.ionio.gr/zSAPN. For every source which does not support the Access Point, a new query is produced using the Bib-1 RDFS and its source description for the supported Access Points. The core of our system which implements the substitution algorithms is built on top of the ICS-Forth RDFSuite (http://athena.ics.forth.gr:9090/RDF/) and for the exploitation of the Bib-1 RDFS we use the RQL query language [5]. For Z39.50 services we use the PHP/YAZ toolkit from IndexData (http://www.indexdata.com/phpyaz).

The homepage of zSAPN is appearing into figure 2 . The current version of zSAPN at http://dlib.ionio.gr/zSAPN gives access to some of the most popular and representative Z39.50 sources world wide. For the formulation of a query, a user has to select sources, search terms, Access Point and substitution policy in case a source does not

\section{Z39.50 Semantic Access Point Network v 1.0}

From the source(s): Select All

$\square$ Library and Archives Canada $\square$ Library of Congress

$\square$ MELVYL $\square$ COPAC

$\square$ Hellenic Academic Libraries Union Catalogue $\square$ National and Kapodistrian University of Athens

$\square$ Technological Educational Institute of Patras $\square$ University of Crete

$\square$ University of Cyprus

Search for:

In case where a source does not support the requested Access Point,

Select how to substitute it.

$\bigcirc$ Broad Substitution $\odot$ Narrow Substitution $\bigcirc$ NO Substitution

Detailed Results $\odot$ The minimal set only

Fig. 2. zSAPN first page for starting a session 
support the selected Access Point. As we see in figure 2, zSAPN offers also an option to broadcast the query directly to the selected sources as it is, without substitution, and to leave the sources reply with their default behaviour.

For the clarification of the broad substitution policy let's consider the following example. We assume that we are searching for the proceedings from the IEEE's conferences and only these. It is worth mentioning that we are not interested in other types of IEEE's editions such as technical reports, technical standards etc. Continuing, we select the Z39.50 sources of the Library of Congress, the COPAC Academic \& National Library Catalogue (from United Kingdom) and the University of Crete Library (from Greece). The most appropriate Access Point for our query is the $A u$ thor-name-conference according to the Bib-1 specification, which is rarely available from the majority of the known meta-search or local systems.

Table 2. Results summary from zSAPN broad substitution search response

\begin{tabular}{|l|c|l|}
\hline \multicolumn{1}{|c|}{ Source } & Hits & \multicolumn{1}{c|}{ Query } \\
\hline COPAC & 2810 & $\begin{array}{l}\text { Author-name-conference_1006 = IEEE } \\
\text { The source supports the Access Point. }\end{array}$ \\
\hline Library of Congress & 1714 & $\begin{array}{l}\text { Author-name-conference_1006 = IEEE } \\
\text { From the Access Point Substitution: }(t p=1) \\
--T h e \text { Minimal Set is: } \\
\text { Author-name_1003, Name-conference_3 }\end{array}$ \\
\hline University of Crete & 344 & $\begin{array}{l}\text { Author-name-conference_1006 = IEEE } \\
\text { From the Access Point Substitution: }(t p=0.33) \\
-- \text { The Minimal Set is: } \\
\text { Author-name_1003 }\end{array}$ \\
\hline
\end{tabular}

Table 2 depicts the part of zSAPN response page presenting the summary of the search results of the example query. The COPAC source supports the query thus no substitution is required. The Library of Congress source does not support the Access Point $^{2}$ Author-name-conference so the system substitutes the unsupported Access Point with the Boolean AND combination of the supported Access Points Authorname and Name-conference. As the system replies for the Library of Congress substitution $t p=1$. Finally, for the source of the University of Crete, zSAPN substitutes the unsupported Access Point with the supported Author-name with $t p=0.33$. We observe the different substitutions for the sources of the Library of Congress and the University of Crete, influencing the differences into their sets of supported Access Points.

Before analyzing the comparison of the semantics similarity between the requested Access Point and the Access Points resulted from the broad substitutions in the previous example, let's examine the semantics of the used Access Points, as depicted in figure 1. As we already presented in section 2.3, the Author-name-conference has equivalent semantics with the intersection (Boolean AND combination) of its direct ancestors Author-name and Name-conference. The semantic set of data fields of the Access Point Author-name consists of more fields than the Author-name-conference but less than the Name Access Point.

\footnotetext{
${ }^{2}$ According to the document "LC Z39.50/SRW/SRU Server Configuration Guidelines" at: http://www.loc.gov/z3950/lcserver.html
} 
Concluding the comparison between the semantics of zSAPN's broad substitutions and the semantics of the requested access point, we observe that the substitution for the Library of Congress produces equivalent results with the requested Access Point and its answer has the same precision as the COPAC's answer which supports the Access Point. For the University of Crete, we receive an answer with similar semantics, but with less precision. In this way we manage to exclude records having as subject the conferences of IEEE, although the answer still contains also other types of editions of IEEE (e.g. technical standards, etc.).

Table 3. Resulting hits without substitution and with broad substitution

\begin{tabular}{|l|c|c|}
\hline \multicolumn{1}{|c|}{ Source } & No Substitution & Broad Substitution \\
\hline COPAC & 2810 & 2810 \\
\hline Library of Congress & 8081 & 1714 \\
\hline University of Crete & Error: Unsupported attribute combination & 344 \\
\hline
\end{tabular}

Ending the zSAPN presentation for the broad Access Point substitution, we compare the number of hits resulted from the answers using zSAPN with the no substitution and with the broad substitution options. As we observe from Table 3, for COPAC it makes no difference due to the support of the Access Point from the source. The Library of Congress does not inform the user that the Access Point is not supported and does not also give any further information concerning the arbitrary substitution of the unsupported Access Point. Moreover, for the Library of Congress, we see a substantial difference in the number of matching records, about 4.5 times more hits approximately. We remind that the substitution for the Library of Congress produces equivalent semantics with the original request $(t p=1)$. For the University of Crete, the user receives a similar answer with less precision $(t p=0.33)$ instead of a query failure.

The second substitution policy narrows the semantics of the requested Access Point. Let's consider again another example for the presentation of the narrow substitution policy. We assume that we are searching the Library \& Archives Canada source, for all the metadata records containing the term "Malinowski" as either Author, or Subject, or in the Title. The most appropriate Access Point from the bib-1 Access Points set is the Author-Title-Subject, which is also rarely offered for use.

Table 4 depicts the part of zSAPN response page presenting the search results summary from the Library \& Archives Canada source. This version of the summary results table contains in the last column both the initial and the final optimized minimal set of Access Points for the substitution. We note that the source does not support the Access Point Author-Title-Subject and we select the narrow substitution policy. zSAPN substitutes the unsupported Access Point Author-Title-Subject with the Boolean $O R$ combination of the supported Access Points Title, Subject and Author-name thus avoids the query failure from the source.

As we already mentioned, for both substitution policies the final set of Access Points for substitution is the minimal set of supported Access Points having the nearest semantics to the unsupported Access Point. We observe in table 4 that the initial set for substitution of the unsupported Access Point consists of the Title, Subject, 
Table 4. Results summary from zSAPN narrow substitution search response

\begin{tabular}{|c|c|l|}
\hline Source & Hits & \multicolumn{1}{c|}{ Query } \\
\hline & & Author-Title-Subject_1036 = Malinowski \\
& & From the Access Point Substitution: $($ tr=0.95) \\
& & - The AP could be substituted with the AP(s): \\
Library and Archives Canada 52 & Title_4, Subject_21, Author-name_1003, \\
& & Author-name-corporate_1005 \\
& & Author-name-personal_1004 \\
& & Author-name-conference_1006 \\
& & --The Minimal Set is: \\
& Title_4, Subject_21, Author-name_1003 \\
\hline
\end{tabular}

Author-name-corporate, Author-name-personal, Author-name and Author-nameconference, while the minimal set consist of the Access Points Title, Subject and Author-name. Whereas the Access Points Author-name-corporate, Author-namepersonal and Author-name-conference are subsets of the Access Point Author-name, see figure 1, their semantics are already included into the Access Point Author-name. Therefore, the optimization step excludes the Access Points Author-name-corporate, Author-name-personal and Author-name-conference from the minimal set of the narrow substitution policy.

In the previous example, the user receives an answer with similar results to the original query and avoids the query failure. We remind that the Library and Archives Canada source fails the query when it does not support a requested Access Point, like the Author-Title-Subject. Having failed the query, the user does not retrieve results while he is notified that the Access Point is not supported. Thereafter, the user has two alternatives in order to search the Library and Archives Canada source: either to broad the semantics using a more general Access Point like Any, or to replace the Access Point with the union of other Access Points with narrower semantics, similar to what zSAPN would do. The first, the easiest one, will increase the results by approximately $57 \%$ due to receiving 30 additional non relevant matching records, while the second requires a deep knowledge of the semantic relationships of the Access Points and the available search services from the source.

\section{Conclusions and Future Research}

We found that semantics based substitution methods could really improve the effects from the unsupported Access Points when meta-searching metadata repositories behind query interfaces with abstract Access Points. The current implementation of the zSAPN system, in the context of the Z39.50 protocol, improves the search consistency and lowers the query failures exploiting the semantic information of the Access Points from an RDFS description. Moreover, zSAPN substitutes the unsupported Access Point with a set of others whose proper combination either broadens or narrows the semantics of the unsupported Access Point, while evaluates the modification on the precision or the recall for the original query respectively.

The proposed substitution policies enable the meta-search system, or any other mediator, to decide how to modify, if it is necessary, the semantics of an unsupported query prior to initiating the search requests. In addition, a source using the zSAPN 
underlying methodology could expand its functionality instead of making arbitrary or general substitutions, as is the case of the Library of Congress, and also better schedule the retrieval process using the taxonomic precision and recall values. Finally, the Bib-1 Access Points RDFS description could be a basis for the deployment of the library community search primitive semantics to the Semantic Web [2].

A number of interesting points are derived from this work for future development and research. First, we have to extent the measures so that the evaluation of the precision and recall influence additionally the importance of the constitutional fields of the Access Point. Moreover, we have to evaluate the overall modification of the recall and the precision when a query contains more than one unsupported Access Points. Up to this point we have only studied the subset relationship between the Access Points. A number of questions remain to be answered like: How tight are the subset relationships and what parts of these semantics are used in practice? Is it possible to automate the process of the Access Point substitution so that the system will be able to decide the best substitution policy under some criteria?

Another motivating study would be the application of our methodology and algorithms to the corresponding concepts of the Search and Retrieve Web Services (SRW) protocol [9] and to measure the degree of improvement. An essential point for the deployment of SRW would be the creation of a meta-RDF schema expressing the correlations of underlying semantics contained in every context set defined in the protocol.

\section{References}

1. ANSI/NISO: Z39.50 Information Retrieval: application service definition and protocol specification (approved May 10, 1995)

2. Berners-Lee, T., Hendler, J., Lassila, O.: The Semantic Web. Scientific American (May 2001)

3. Beacom, M., et al. (eds.): Anglo-American Cataloguing Rules. ALA, Chicago (2004)

4. Dellschaft, K., Staab, S.: On How to Perform a Gold Standard Based Evaluation of Ontology Learning. In: Cruz, I., Decker, S., Allemang, D., Preist, C., Schwabe, D., Mika, P., Uschold, M., Aroyo, L.M. (eds.) ISWC 2006. LNCS, vol. 4273, pp. 228-241. Springer, Heidelberg (2006)

5. Karvounarakis, G., Alexaki, S., Christophides, V., Plexousakis, D., Scholl, M.: RQL: A Declarative Query Language for RDF. In: 11th International World Wide Web Conference (WWW 2002), Honolulu, Hawaii, USA (2002)

6. Maedche, A., Staab, S.: Measuring similarity between ontologies. In: Gómez-Pérez, A., Benjamins, V.R. (eds.) EKAW 2002. LNCS (LNAI), vol. 2473. Springer, Heidelberg (2002)

7. Sfakakis, M., Kapidakis, S.: A Semantics-Based Graph for the Bib-1 Access Points of the Z39.50 Protocol. In: Gonzalo, J., Thanos, C., Verdejo, M.F., Carrasco, R.C. (eds.) ECDL 2006. LNCS, vol. 4172, pp. 445-448. Springer, Heidelberg (2006)

8. Sfakakis, M., Kapidakis, S.: A Semantics-Based Substitution for Unsupported Z39.50 Bib1 Access Points. In: Proceedings of the 2nd International Conference on Digital Information Managemant (ICDIM 2007), Lyon, France, October 28-31 (2007)

9. Sanderson, R.: A Gentle Introduction to SRW (2004), http://srw. cheshire3.org/docs/introduction.html

10. W3C: Resource Description Framework Schema Specification 1.0. W3C Candidate Recommendation (March 27, 2000), http://www.w3.org/TR/2000/CR-rdf-schema-20000327/

11. ZIG: Attribute Set BIB-1 (Z39.50-1995): Semantics, http: / / www. loc.gov/z3950/agency/bib1.html 